\title{
Paper
}

\section{Operating Method of Human Collaborative Robot using Self-Capacitance Proximity and Tactile Sensor}

\author{
Satoshi Tsuj* ${ }^{* \dagger}$ Member, $\quad$ Teruhiko Kohama $^{\dagger}$ Non-member
}

(Received October 3, 2018, revised December 19, 2018)

\begin{abstract}
In recent years, human collaborative robots (HCRs) working in a same place with people has become more attractive. We have proposed a self-capacitance proximity and tactile skin sensor for HCRs. The structure of the sensor is simple and consists of two electrodes $\left(\mathrm{E}_{1}\right.$ and $\left.\mathrm{E}_{2}\right)$ and an elastic body. The measurement system is also simple because only the self-capacitance measurement is used to detect objects both before and after contact. In this paper, we developed a ten-sensor system covering a whole robot surface. This sensor can detect objects before contact, and can detect pressure and positions after contact. In addition, we propose an operating method using the sensor for the safety and workability of HCRs. The proposed operating method including the sensor may be a useful system for HCRs.
\end{abstract}

Keywords: Operating method of robot, Proximity sensor, Tactile sensor, Capacitance measurement

\section{Introduction}

Most current robots are industrial robots that work mainly in factories. Many of the working spaces of these industrial robots are separated from people by fences to ensure safety. Recently, human collaborative robots (HCRs) working in the same place with people has become more attractive. Workability and space-saving can be expected when HCRs work in a same space as people. HCRs require various sensors for workability and safety in collaboration with humans. Among them, skin sensors covering the entire robot are important for detecting contact conditions with objects.

An objective of this study was to establish a simplified system of proximity and tactile skin sensors for HCRs. Figure 1 shows a goal image of the proposed sensor system on an HCR that is working with humans. The skin sensor constantly measures the state near the robot surface, and uses that information to avoid contact with objects including humans. Thus, the skin sensor increases the workability and safety of HCRs.

In recent years, several types of tactile skin sensors for robots have been developed [1] [5]. Using optical elements to detect contact pressure has been proposed [1]. A skin sensor using LED, photo transistor, and silicone cap can detect three components of an applied force from contact. A method of low wiring and high response speed has been proposed [2]. A tactile sensor using a conductive sheet can detect a contact pressure and a center point. Using capacitance measurement has been proposed [3] [4]. A tactile sensor using two types of urethane foam and electrodes can

\footnotetext{
* Corresponding: tsuji@fukuoka-u.ac.jp

$\dagger$ Department of Electrical Engineering, Fukuoka University, 8-19-1

Nanakuma, Jonan-ku, Fukuoka 814-0180, Japan
}

detect contact force and contact area [3]. Triangular modules capacitance skin sensors can detect pressure [4]. These sensors have a measurement IC in the sensor, and can be connected to other sensors to cover a large area of robot surface. Additionally, a stretchable skin sensor, which uses conductive rubber by electrical impedance tomography [5], can detect pressure, and may be set on a joint of a robot. These skin tactile sensors can detect an object after contact. In addition, collaborative robots using joint torque sensors have been on sale [6]. However, these tactile sensors and the robots have difficulty detecting an object before a robot touches it, and avoiding an object before contact.

HCRs need to avoid contact with objects using proximity skin sensors, and proximity sensors for robots have been proposed [7] [9]. A proximity sensor using optical elements for low wiring and high response speed has been proposed [7]. The proximity sensor can detect an object and its center point before contact. A proximity sensor using capacitance measurement has been proposed [8] [9]. In

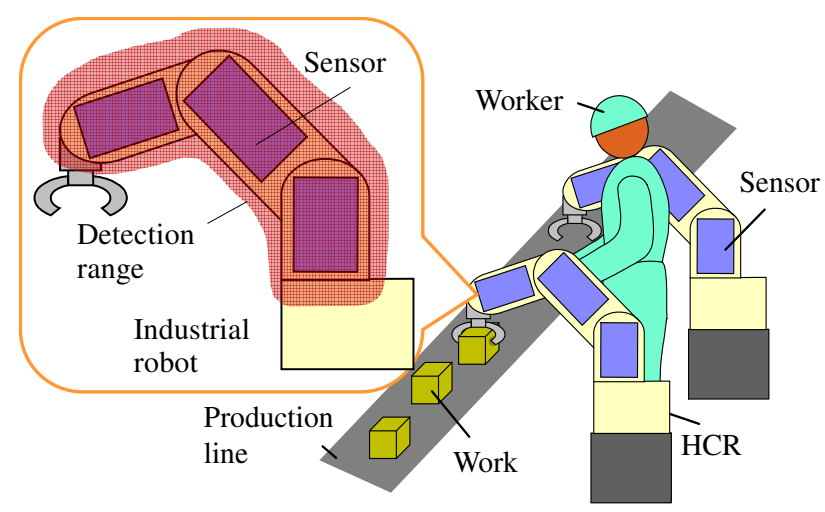

Figure 1: Goal image of this study. 
addition, a collaborative robot using capacitive proximity sensors has been on sale [10]. However, these proximity sensors cannot detect a contact condition.

To detect an object both before and after contact, proximity and tactile sensors have been proposed [11] [13]. A robotic skin sensor using an optical element can detect an object before and after contact [11]. However, it may be difficult to separate a proximity state and a contact state because proximity and contact information are obtained from the same information. Also, a tactile and proximity sensors using mutual capacitance measurement can detect an object at proximity range and on a contact [12] [13]. However, a mutual capacitance measurement requires binary electrodes, and the structural flexibility of the sensor is thereby degraded.

We have developed a self-capacitance proximity and tactile sensor [14]. The self-capacitance measurement requires a single electrode. The sensor structure is simple because it consists of two measurement electrodes and an elastic body. The measurement system is also simple because only the self-capacitance measurement is used for the measurement data. The sensor on a robot can detect objects both before and after contact.

Techniques to operate HCRs using sensors have been proposed [1] [6] [8] [10]. Using a tactile or joint torque sensor, a method of stopping robot movement by contact, and operating the robot by pressure from touch has been proposed [1] [6]. However, a robot with a sensor cannot detect an object until touch. In the case of using proximity sensors on a robot, a method of stopping or avoiding the robot when an object approaches the sensor on a surface of the robot has been proposed [8] [10]. However, operation of peripheral devices and the functional operation of the robot is not considered. In addition, an operation system using proximity sensor has been proposed [9]. However, the safety is inadequte compared with a three-position switch because it is difficult to detect a contact condition. On the other hand, manual operation of a industrial robot typically uses a teach pendant; however, a worker needs expert knowledge to operate a robot using the teach pendant.

In this paper, we propose an operating method for HCRs using the proposed self-capacitance proximity and tactile sensor. We developed a ten-sensor system suitable for robot surfaces [15]. This sensor can detect an object before contact, and can detect pressure and its position after contact. In this proposed method, HCRs with sensors can be operated intuitively from sensor information without a teach pendant. The novel point of the operating method is a griping motion (push two directions on the sensors) for peripheral device operations and robot function operations, in addition to the conventional operation.

Section II of this paper presents a proposed principle of a proximity and tactile method. Section III describes a prototype sensor design. Section IV provides an evaluation of results and discussion. Section V presents the conclusions. (a)

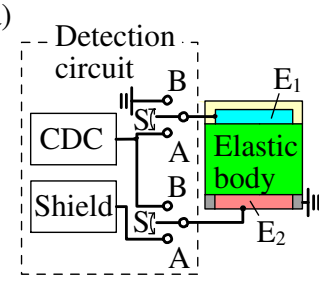

(b)

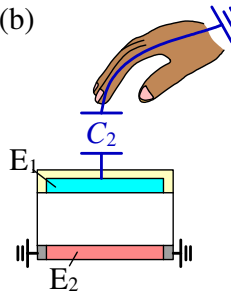

(c)

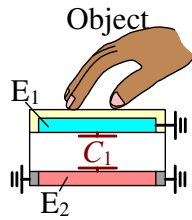

Figure 2: Measurements by the proposed method.

\section{Principle}

In this proposed method [14], only the self-capacitance measurement is used to detect an object both before and after contact. A self-capacitance is a capacitance between single measurement electrode and ground, and it is easy to change the size and shape of a sensor. Figure 2 (a) shows a schematic diagram of the proposed sensor. The sensor consists of an upper electrode $\left(E_{1}\right)$, lower electrode $\left(E_{2}\right)$, and an elastic body. A self-capacitance between $E_{1}$ or $E_{2}$ and ground is measured by switching between $E_{1}$ and $\mathrm{E}_{2}$. Figures 2 (b) and (c) show an image of a proximity and contact measurement of the sensor. In Fig. 2(b), $E_{1}$ is connected to a capacitance digital converter (CDC), and the self-capacitance $\left(C_{2}\right)$ of $\mathrm{E}_{1}$ is measured (connection $\mathrm{A}$ ). Here, $E_{2}$ is connected to a shield. In Fig. 2(c), $E_{2}$ is connected to CDC, and a self-capacitance $\left(C_{1}\right)$ between $\mathrm{E}_{2}$ and $\mathrm{E}_{1}$, which is connected to ground, is measured (connection B). In connection A, when the object approaches the sensor, $C_{2}$ changes according to both the permittivity of the object and the distance between the sensor and the object. Thus, the sensor can detect the object using $C_{2}$ before contact. Furthermore, the sensor can distinguish a material in the object using $C_{2}$ upon contact. In connection $\mathrm{B}$, when an object approaches the sensor, $C_{1}$ does not change because $\mathrm{E}_{1}$ is connected to the ground. When the object touches the sensor, $C_{2}$ changes according to an indentation, which is equal to a distance between $\mathrm{E}_{2}$ and $\mathrm{E}_{1}$. Thus, the sensor can detect the contact condition using $C_{1}$. Therefore, the sensor can detect an object before and after contact using the self-capacitance measurement.

\section{Prototype Sensor}

The structure of the prototype sensor I shown in Figs. 3(a) and 3(b) is the ten-sensor prototype [15]. In our design, $\mathrm{E}_{1}$ is large to increase sensitivity at a proximity range, and $E_{2}$ is small to realize a high spatial resolution on contact. The size of the sensors is $150 \times 75 \mathrm{~mm}$ (sensors I, III, IX, and $\mathrm{X}), 90 \times 60 \mathrm{~mm}$ (sensors II and IV), $60 \times 70 \mathrm{~mm}$ (sensors V and VII), and $140 \times 80 \mathrm{~mm}$ (sensors VI and VIII) from the attachment to a robot arm (DENSO, VS-050). Here, sensors I, III, VI, VIII, IX, and X consist of $2 \times 3$ electrodes for $E_{2}$ and one electrode for $E_{1}$. Sensors II, IV, V, and VII consist of $2 \times 2$ electrodes for $E_{2}$ and one electrode for $E_{1}$. A urethane gel (thickness: $2 \mathrm{~mm}$, hardness: 0 (ASKER C), UG in Fig. 3(a)) is set between $E_{1}$ and $E_{1}$. A silicone sheet (thickness: $0.1 \mathrm{~mm}, \mathrm{Si}$ in Fig. 3(a)) is placed on the sensor surface to isolate $E_{1}$ from an object. A grounded electrode $(\mathrm{G})$ and shield electrodes $(\mathrm{S})$ are set on a back of the sen- 


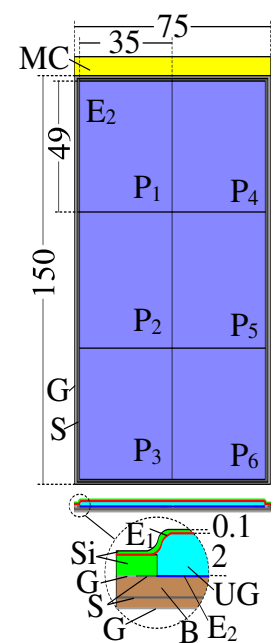

(a)

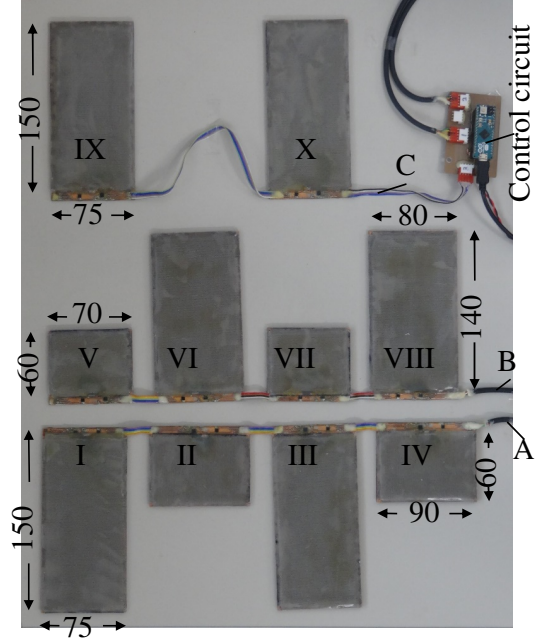

(b)
Figure 3: Schematic diagram of the proposed sensor. $E_{1}$ and $\mathrm{E}_{2}$ : electrodes, UG: Urethane gel, Si: Silicone, S: Shield, G: Ground, B: base, MC: Measurement circuit. (Dimensions in $\mathrm{mm})$.

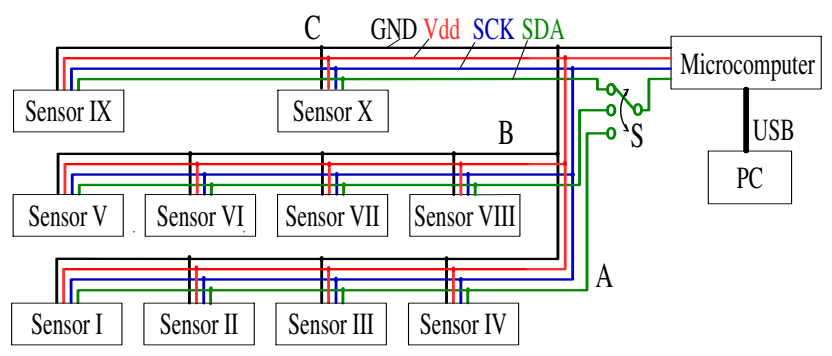

Figure 4: Measurement system.

sor and around $\mathrm{E}_{2}$ to reduce electrical noise from the robot. Self-capacitances are measured by a measurement circuit (MC), which is attached to a side of the sensor to reduce electrical noise from wiring. MC consists of a capacitance measurement IC (Analog Devices, AD7147 is CDC), and an analog switch that changes the electrical condition of $\mathrm{E}_{1}$. Connections A and B are continuously switched using a microcontroller and analog switches.

Figure 4 shows the measurement system. Measured data (16 bit) by each AD7147 (slave device) are sent to the microcomputer (master device) by an $\mathrm{I}^{2} \mathrm{C}$ serial interface bus. The measured data are sent to a $\mathrm{PC}$ from the microcomputer by USB. The $\mathrm{I}^{2} \mathrm{C}$ serial interface bus uses four lines, which are serial data line (SDA), serial clock line (SCL), power line (Vdd) and ground line (GND). Four sensors can be connected with one $\mathrm{I}^{2} \mathrm{C}$ line because $\mathrm{AD} 7147$ has four $\mathrm{I}^{2} \mathrm{C}$ device addresses. The sensor system is divided into three parts (A-C). Each parts' devices are connected to the master device by each SDA and common lines. Thus, this sensor system needs six wires, which are three SDA, one SCL, one Vdd, and one GND line. Each SDA is switched by a multiplexer continuously to send measurement data. Furthermore, the sensor can be increased on the same $\mathrm{I}^{2} \mathrm{C}$ line. Thus, wiring can be reduced. A unit of measurement values indicates $\mathrm{A} / \mathrm{D}$ converted digital values (digit).

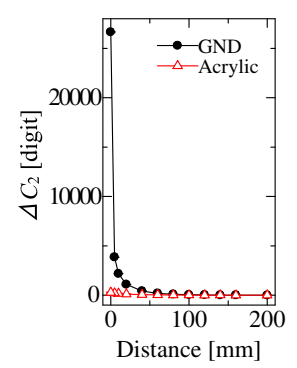

(a)

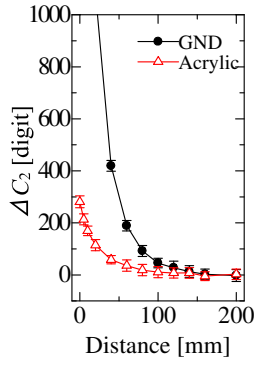

(b)

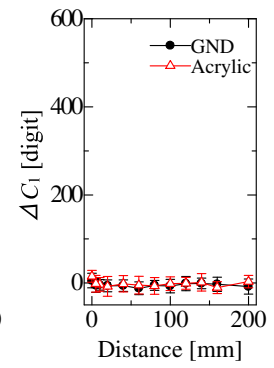

(c)
Figure 5: Measurement results at proximity range.

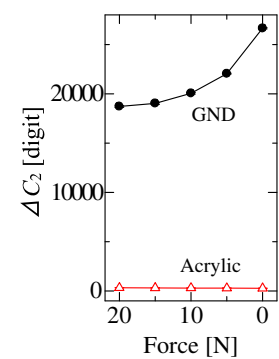

(a)

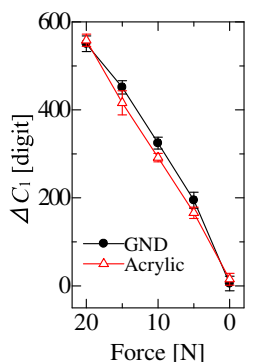

(b)

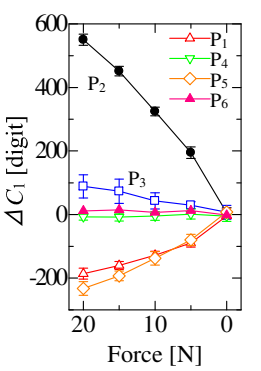

(c)
Figure 6: Measurement results on contact.

\section{Results and Discussion}

4.1 Proximity and Contact Measurements The proposed sensor can detect an object both before and after contact [14]. We evaluated the proximity and contact measurements of the prototype sensors. Objects are grounded conductors (GND), which are human models, and acrylic. The size of the object is $30 \times 30 \mathrm{~mm}$. The object is set on $\mathrm{P}_{2}$ of sensor I. The distance between the sensor and the object is changed from 0 to $200 \mathrm{~mm}$ by the robot arm. The contact condition is measured by the force gauge. Figure 5(a) shows the $\Delta C_{2}$ of sensor I at proximity range, Fig. 5(b) shows the enlarged view of Fig. 5(a), and Fig. 5(c) shows $\Delta C_{1}$ of $\mathrm{P}_{2}$ on sensor $\mathrm{I}$ at proximity range. Here, $\Delta C_{1}$ and $\Delta C_{2}$ are the variations in $C_{1}$ and $C_{2}$, respectively. $\Delta C_{2}$ changes according to the distance in Figs 5(a) and (b). Thus, the sensor can detect an object before contact using $\Delta C_{2}$. This sensor can detect an object (GND) within approximately $100 \mathrm{~mm}$. Here, proximity detection distance of an object depends on an electrical characteristic of the object and the overlap between the sensor and the object. $\Delta C_{1}$ does not change at non-contact, and $\Delta C_{1}$ changes after contact. Thus, the sensor can detect the contact using $\Delta C_{1}$ [14]. Figure 6(a) shows $\Delta C_{2}$ on contact, Fig. 6(b) shows $\Delta C_{1}$ of $\mathrm{P}_{2}$ on contact, and Fig. 6(c) shows $\Delta C_{1}$ of each $\mathrm{E}_{2}$ on contact when the object is GND. $\Delta C_{2}$ in Fig. 6(a) change according to the permittivity of the object. Thus, the sensor can discriminate between the grounded conductor and other objects. $\Delta C_{1}$ changes according to pressure. Thus, the sensor can detect approximate pressure and its position [14].

Figure 7 shows the $\Delta C_{1}$ and $\Delta C_{2}$ of all sensors when the object was a hand [15]. Fig. 7(a) shows the position of the hand and the sensors. The hand approached the sensor (Fig. 7 B, C and E), a finger pushed the sensor (Fig. 7 D), and 

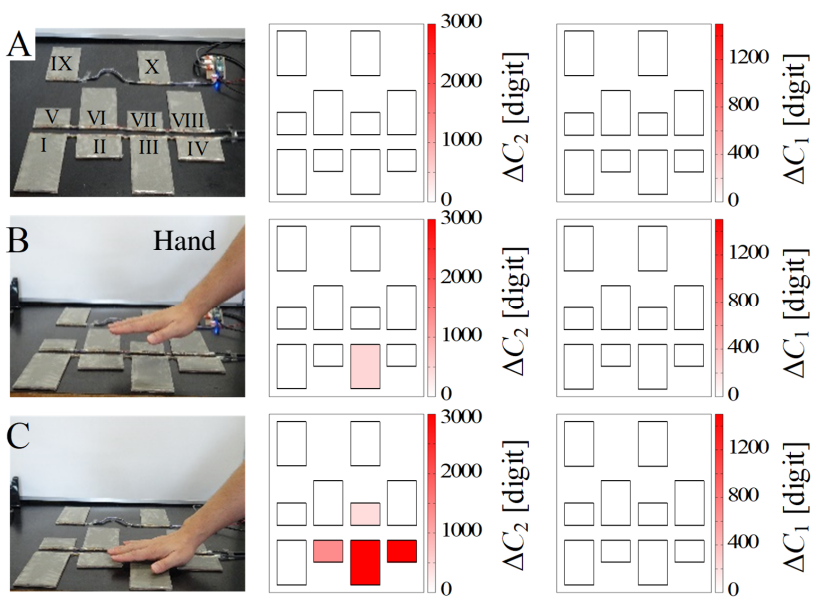

$\mathrm{D}$
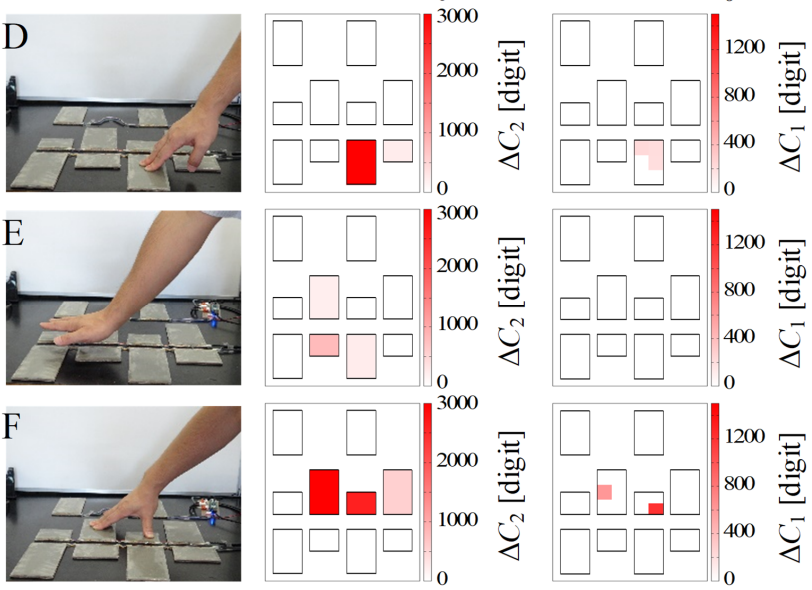

(a)

(b)

(c)

Figure 7: Measurement results of the proposed sensors.

two fingers pushed the sensor (Fig. 7 F). Figure 7(b) shows $\Delta C_{2}$, and Fig. 7 (c) shows $\Delta C_{1} . \Delta C_{1}$ and $\Delta C_{2}$ are denoted in white in Fig. 7 when values are less than \pm 3 standard deviations (SDs) lower than each value of air. In addition, the $\Delta C_{2}$ in Fig. 7(b) shows 3000 digits when the values are more than 3000 digits, and $\Delta C_{1}$ in Fig. 7(c) shows 1500 digits when the values are more than 1500 digits. In Fig. 7 (b), $\Delta C_{2}$ changes according to a distance at non-contact points (B, C, and E). Thus, the sensor can detect an object before contact (proximity range). In Fig. $7(\mathrm{c}), \Delta C_{1}$ changes according to pressure and an object position when the object was pushed ( $\mathrm{D}$ and $\mathrm{F}$ ). In addition, $\Delta C_{1}$ does not change without contact (A, B, C, and E). Thus, the sensors can detect the pressure and the object position on contact. It takes approximately $61 \mathrm{~ms}$ to obtain one complete cycle of measurements (ten-sensor) with a data transmitting time from a microcomputer to PC. It takes approximately $35 \mathrm{~ms}$ to obtain one complete cycle of measurements (ten-sensor) without the data transmitting time from the microcomputer to PC.

\subsection{2 Operating Method of HCRs using the Proto-} type Sensor We propose an operating method of HCRs using sensors on a robot. The sensors are attached to a surface of the robot arm (Fig. 8). The operation is to improve safety and workability of HCRs because people and robots

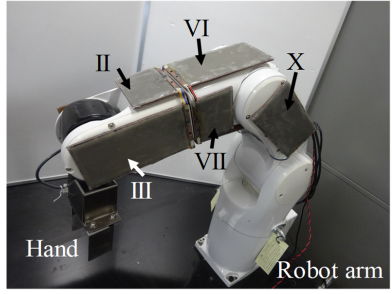

(a)

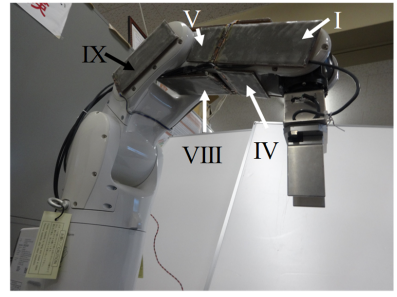

(b)
Figure 8: Proposed sensors on the robot arm surface. (a) Top view. (b) Under view.

(a)

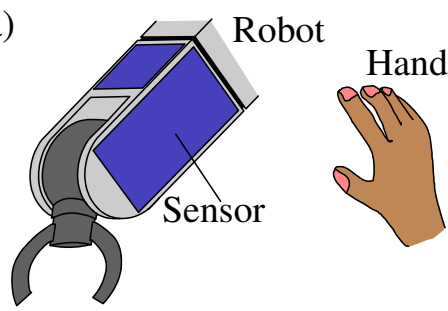

(b)

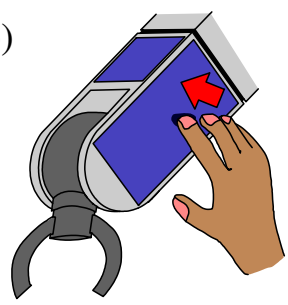

(c)

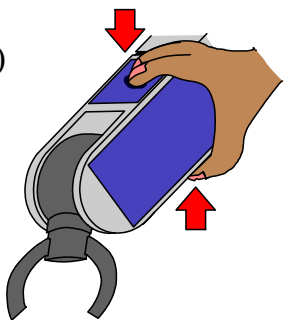

Figure 9: Image of the proposed operation method.

work in the same space without safety fences. The manual operation of a robot typically uses a teach pendant. However, professional knowledge is required for a worker to operate the robot with a teach pendant. To operate a robot intuitively, we use measurement data of the sensors on the robot surface. Figure 9 shows an image of the operating method using the sensors. The robot speed is reduced or stopped when an object approaches the working robot (Fig. 9(a)). Thus, sensors can improve safety and functionality of a robot because the robot avoids unnecessary contact with objects, including humans. When the hand of a worker pushes one direction of the sensor, the robot moved based on pressure and position (Fig. 9(b)). The operation is performed on constant pressure like a three position switch to safety. When the hands grip the robot (push two directions), the worker operates the peripheral devices and functions of the robot (Fig. 9(c)). Thus, the proposed sensor can increase the functionality of a robot.

We evaluated the measurement of the sensors on the robot 

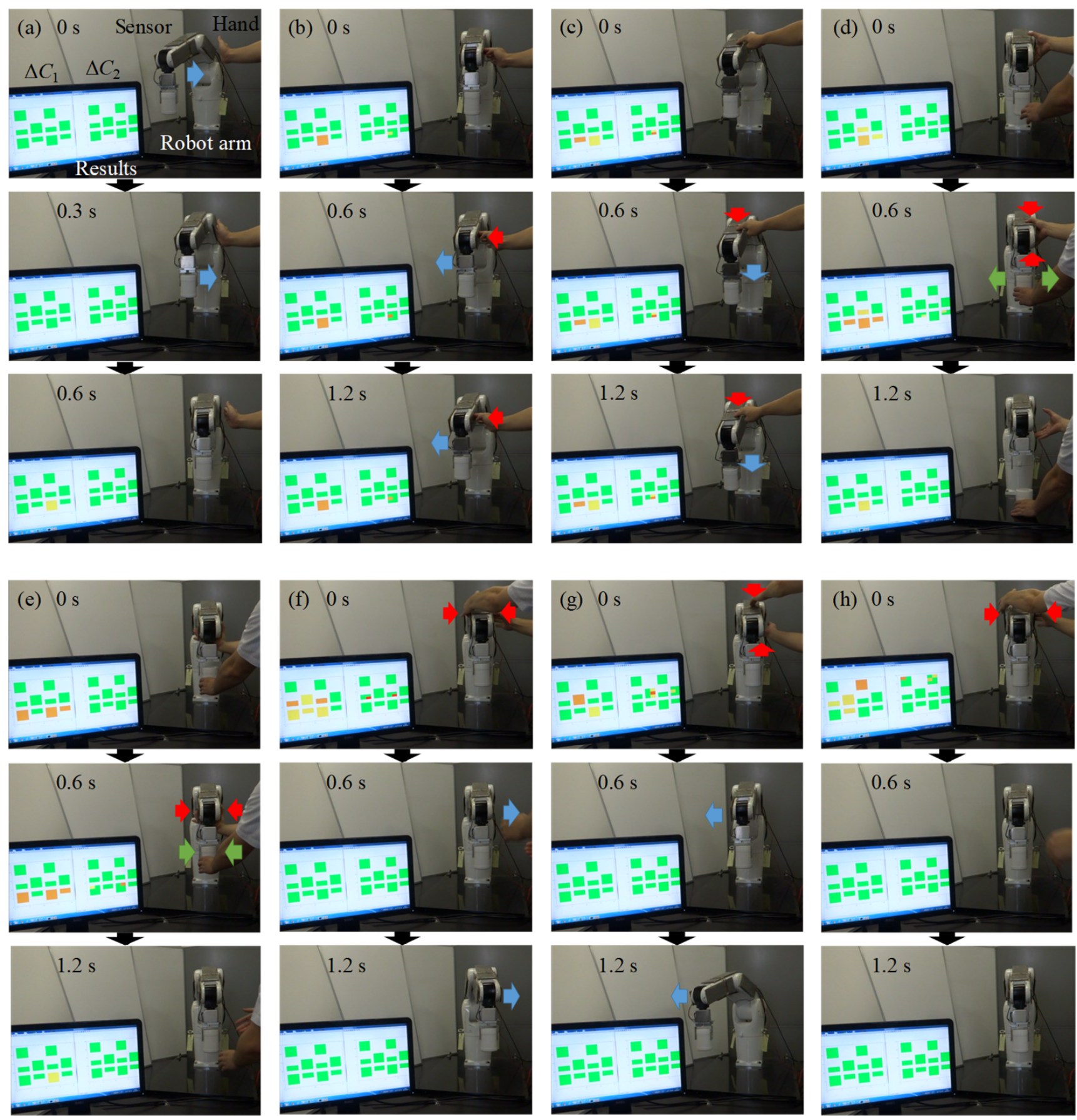

Figure 10: Measurement and operation results of the proposed sensors on the robot arm surface.

arm surface, and the operation of the robot using the measurement results. In this study, we operated the robot as below.

1. When the sensor detects an object before contact, the robot is stopped until the object leaves.

2. When the sensor detects one-directional pressure of an object, the robot is moved according to the pressure direction.

3. When the sensor detects a facing pressure by hands at the same time, a robot hand is opened in the case of sensors II and IV, the robot hand is closed in the case of sensors I and III, the moving speed of the robot slows in the case of sensors V and VII, the speed of the robot is normal in the case of sensors VI and VIII, and the robot is paused until a worker releases the stop in the case of sensors IX and X.

Figure 10 shows measurement and operational results. A monitor in Fig. 10 projects the $\Delta C_{1}$ and $\Delta C_{2}$ of each sensor. $\Delta C_{1}$ and $\Delta C_{2}$ are denoted in green on the monitor when values are less than \pm 3 SDs lower than each value of air. A red arrow shows the pressure direction by a hand. A blue 
arrow shows a moving direction of the robot. Figure 10(a) shows that the robot approaches the hand, and the robot stops when the $\Delta C_{2}$ of sensor III is changed, and the sensor (III) detects the object before contact. Figure 10(b) shows that the hand pushes the sensor (III), the robot is moved according to a pressure direction (left side) when the $\Delta C_{1}$ of sensor III is changed. Also, Fig. 10(c) shows that the hand pushes sensor (II), and the robot is moved according to a pressure direction (downside) when the $\Delta C_{1}$ of sensor II is changed. Fingers push sensors II and IV (Fig. 10(d)). The robot hand is opened when the $\Delta C_{2}$ of sensors II and IV is changed at the same time. The fingers push sensors I and III (Fig. 10(e)). The robot hand is closed when the $\Delta C_{2}$ of sensors I and III is changed at the same time. The fingers push sensors V and VII (Fig. 10(f)). The robot speed slows when the $\Delta C_{2}$ of sensors $\mathrm{V}$ and VII are changed at the same time. The fingers push sensors VI and VIII (Fig. 10(g)). The robot speed set normal when the $\Delta C_{2}$ of sensors VI and VIII are changed at the same time. Finally, the fingers push sensors IX and X (Fig. 10(h)). The robot is paused until a worker releases the stop when the $\Delta C_{2}$ of the sensors IX and $X$ are changed at the same time. It was confirmed that the robot can be directed by these operations in real time. In addition, operations can be changed freely according to a setting, and operational functions can be added. Thus, the proposed sensor can increase safety because a robot can avoid unexpected contact with objects including humans using sensor information. In addition, the sensor can increase the functionality of a robot because the robot can be operated using the sensor without a teach pendant.

\section{Conclusions}

In this paper, we proposed an operating method for HCRs using a self-capacitance proximity and tactile skin sensor. This proposed sensor consists of two electrodes $\left(E_{1}\right.$ and $\left.E_{2}\right)$ and elastic body. We produced the ten-sensor system on the same $\mathrm{I}^{2} \mathrm{C}$ bus line to cover the robot surface. The sensors on the robot surface can detect an object before and after contact. In addition, we proposed the following operation method.

1. When the sensor detects an object before contact, the robot is stopped until the object leaves.

2. When the sensor detects one-directional pressure from an object, the robot is moved according to the pressure direction.

3. When the sensor detects the facing pressure of an object, the robot hand is opened and closed, the robot speed is controlled, and the robot is paused until a worker releases the stop. These operations depend on pushing positions.

Therefore, we think that the method is intuitive for the operation of HCRs without a teach pendant. The proposed sensors are thus applicable to tactile skin sensors for HCRs.

\section{Acknowledgment}

This work was supported in part by funds (No. 185009) from the Central Research Institute of Fukuoka University.

\section{References}

[1] A. Cirillo, F. Ficuciello, C. Natale, S. Pirozzi, and L. Villani, "A Conformable Force/Tactile Skin for Physical HumanRobot Interaction", IEEE Robotics and Automation Letters, Vol. 1, No. 1, pp. 41-48, 2016

[2] M. Shimojo, T. Araki, A. Ming, and M. Ishikawa, "A HighSpeed Mesh of Tactile Sensors Fitting Arbitrary Surfaces", IEEE Sensors Journal, Vol. 10, No. 4, pp. 822-830, 2010

[3] T. Hoshi, H. Shinoda, "A Sensitive Skin Based on TouchArea-Evaluating Tactile Elements", Proceedings of IEEE Symposium on Haptic Interfaces for Virtual Environment and Teleoperator Systems, 2006, pp. 89-94

[4] P. Maiolino, M. Maggiali, G. Cannata, G. Metta, and L. Natale, "A Flexible and Robust Large Scale Capacitive Tactile System for Robots", IEEE Sensors Journal, Vol.13, No.10, pp. 3910-3917, 2013

[5] H. Alirezaei, A. Nagakubo and Y. Kuniyoshi, "A Tactile Distribution Sensor Which Enables Stable Measurement Under High and Dynamic Stretch", Proceedings of IEEE Symposium on 3D User Interfaces, 2009, pp. 87-93

[6] KUKA LBR iiwa, https://www.robots.com/series/ kuka-collaborative-robot-series, access date: October 3, 2018

[7] H. Hasegawa, Y. Suzuki, A. Ming, K. Koyama, M. Ishikawa, and M. Shimojo, "Net-Structure Proximity Sensor: HighSpeed and Free-Form Sensor With Analog Computing Circuit", IEEE/ASME Transactions on Mechatronics, Vol. 20, No. 6, pp. 3232-3241, 2015

[8] T. Schlegl, T. Kroger, A. Gaschler, O. Khatib, and H. Zangl, "Virtual Whiskers-Highly Responsive Robot Collision Avoidance", Proceedings of IEEE/RSJ International Conference on Intelligent Robots and Systems, 2013, pp. 5373-5379

[9] B. Sekoranja, D. Basic, M. Svaco, F. Suligoj, and B. Jerbic, "Human-Robot Interaction Based on use of Capacitive Sensors”, Procedia Engineering, Vol. 69, pp. 464-468, 2014

[10] BOSCH APAS, https://www.bosch-apas.com/en/ home/, access date: October 3, 2018

[11] D. Hughes, J. Lammie, and N. Correll, "A Robotic Skin for Collision Avoidance and Affective Touch Recognition", IEEE Robotics and Automation Letters, Vol. 3, No. 3, pp. 1386-1393, 2018

[12] H. Lee, S. Chang, and E. Yoon, "Dual-Mode Capacitive Proximity Sensor for Robot Application: Implementation of Tactile and Proximity Sensing Capability on a Single Polymer Platform Using Shared Electrodes", IEEE Sensors Journal, Vol. 9, No. 12, pp. 1748-1755, 2009

[13] D. Goger, H. Alagi, and H. Worn, “Tactile proximity sensors for robotic applications", Proceedings of IEEE International Conference on Industrial Technology, 2013, pp. 978-983. 
[14] S. Tsuji, T. Kohama, "Proximity and Tactile Sensor Using Self-Capacitance Measurement for Human Collaboration Robots", IEEJ Transactions on Sensors and Micromachines, Vol. 138, No. 1, pp. 2-8, 2018

[15] S. Tsuji, T. Kohama, "Development of Whole SelfCapacitance Proximity and Tactile Skin Sensor for Human Collaborative Robot", Proceedings of ICISIP2018, 2018, pp. 368-373

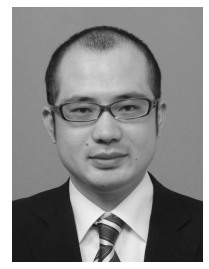

Satoshi Tsuji (Member) received his Ph.D. degree from Saga University, Saga, Japan in 2012. $\mathrm{He}$ is currently an Assistant Professor in the Department of Electrical Engineering, Fukuoka University. His research interests include tactile and proximity sensing systems. He is member of IIAE.

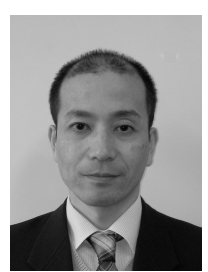

Teruhiko Kohama (Non-member) received his Ph.D. degree from Kyushu University, Fukuoka, Japan in 1999. He is currently an Associate Professor in the Department of Electrical Engineering, Fukuoka University, Japan. He has been active in the fields of power electronics, analog circuits, and sensing systems. 